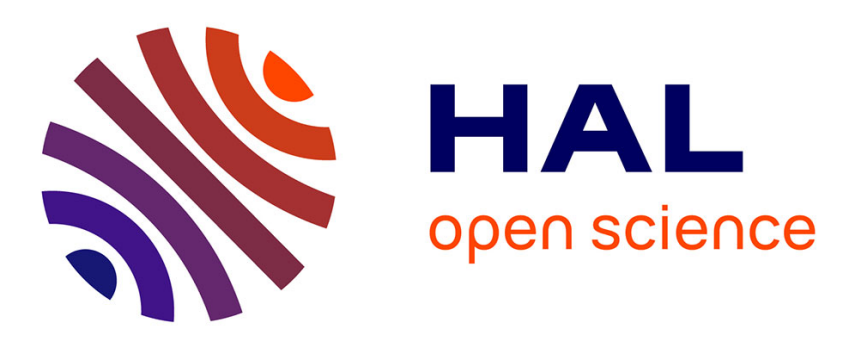

\title{
Préparation d'un fromage semi-dur à partir du lait de chèvre
}

\author{
G. Kalatzopoulos, B. Veinoglou, M. Baltadjieva, C. Alexandropoulos, V. \\ Stamenova, A. Sfiakianos
}

\section{- To cite this version:}

G. Kalatzopoulos, B. Veinoglou, M. Baltadjieva, C. Alexandropoulos, V. Stamenova, et al.. Préparation d'un fromage semi-dur à partir du lait de chèvre. Le Lait, 1983, 63 (629_630), pp.354-361. hal-00928984

\section{HAL Id: hal-00928984 \\ https://hal.science/hal-00928984}

Submitted on 1 Jan 1983

HAL is a multi-disciplinary open access archive for the deposit and dissemination of scientific research documents, whether they are published or not. The documents may come from teaching and research institutions in France or abroad, or from public or private research centers.
L'archive ouverte pluridisciplinaire HAL, est destinée au dépôt et à la diffusion de documents scientifiques de niveau recherche, publiés ou non, émanant des établissements d'enseignement et de recherche français ou étrangers, des laboratoires publics ou privés. 


\title{
Préparation d'un fromage semi-dur à partir du lait de chèvre
}

\author{
par \\ G. KALATZOPOULOS*, B. VEINOGLOU*, M. BALTADJIEVA**, \\ C. ALEXANDROPOULOS*, V. STAMENOVA** \\ et A. SFIAKIANOS*
}

\section{S u m m a ry}

Attempts to make semi hard cheeses from goat's milk were simultaneously tried in Greece and in Bulgaria. Edam cheese technology was adapted to specificities of goat's milk. Characteristics of obtained cheeses were the following:

Moisture $39 \%$; Fat $30 \%$; Proteins 26,5\%; $\mathrm{NaCl} 2,0 \%$.

From results of tasting panel, it is assumed that new cheese will correspond to consumer habits of both countries.

Key words:

Goat's milk - Semi hard cheese - Edam.

* Laboratoire de Technologie laitière, Ecole Supérieure d'Agriculture d'Athènes (Grèce).

** Chaire de Technologie laitière, Institut des Industries alimentaires de Plovdiv (Bulgarie).

*** Technologie Alimentaire, Ministère de l'Agriculture, Lykovrissi - Attiki (Grèce). 


\section{INTRODUCTION}

La place des laits de chèvre et de brebis dans l'économie laitière des pays balkaniques est plus importante que celle du lait de vache. La valorisation de ces laits est essentiellement orientée vers la production de fromage Feta et de yoghourt.

Dans le but de diversifier cette valorisation, nous avons décidé d'étudier la fabrication de nouveaux fromages en adaptant la technologie de fromages connus, généralement obtenus à partir de lait de vache.

Nous avons, en particulier, réalisé des essais de fabrication de fromage de type Edam à partir de lait de chèvre. Ce type de fromage présente l'avantage d'avoir une longue conservation ce qui permettrait de compenser la production saisonnière du lait de chèvre.

\section{MATERIELS ET METHODES}

Le lait de chèvre provenant d'une part de la région de Ionnina au Nord-Ouest de la Grèce et d'autre part de la région de Plovdiv en Bulgarie.

La composition de ce lait a fait précédemment l'objet d'une étude (Veinoglou et al., 1982).

Ce lait était pasteurisé à $72^{\circ} \mathrm{C}$ pendant 15 secondes et transporté ensuite à Athènes à la température de 7,50 $\mathrm{C}$ environ.

La présure utilisée était la présure en poudre Hansen 1 pour 100000 . Nous avons utilisé des ferments mésophiles des Laboratoires Roger (France) composés de Str. Lactis, Str. cremoris et Str. diacetylactis. Les ferments utilisés en Bulgarie étaient constitués d'un mélange similaire mais d'origine bulgare.

Le lait étant pasteurisé, nous y avons incorporé du chlorure de calcium à la dose de $0,1 \%$.

La matière grasse du lait était déterminée selon la méthode acidobutyrométrique de Gerber (Schneider, 1954). Les protéines et le lactose étaient déterminées selon les méthodes de la F.I.L. (F.I.L., 1962, 1964, 1968). L'acidité était exprimée en degrés Dornic.

Lors de chaque fabrication, un fromage entier a été prélevé après $10,30,60,90$ et $120 \mathrm{j}$ d'affinage. Ces fromages étaient ensuite découpés en secteurs circulaires, écroutés puis râpés et conservés en flacons fermés à la température de 2 à $4^{\circ} \mathrm{C}$. 
La matière grasse des fromages était déterminée selon la méthode de Van Gulik (Schneider, 1954). L'azote total, les fractions solubles, l'extrait sec et le taux de $\mathrm{NaCl}$ étaient déterminés par la méthode F.I.L. (F.I.L., 1961, 1962, 1964).

Le coefficient de maturation était défini comme le rapport azote soluble sur azote total. L'analyse des amino-acides a été réalisée selon la méthode de Dimitrov et al. (1976).

Le coefficient de transformation est la proportion du constituant de lait retenu dans le fromage.

La dégustation était réalisée par le personnel de nos laboratoires. La notation était effectuée selon l'échelle proposée par la réglementation française avec un maximum de 20 points (Cogitore, 1972).

Les dégustateurs ont examiné les fromages en tant que nouveaux produits et en tenant compte des habitudes des consommateurs de Grèce et de Bulgarie. Notre but n'était pas de fabriquer un Edam mais d'obtenir un fromage au lait de chèvre répondant aux habitudes de consommation en Grèce et en Bulgarie.

$10 \mathrm{j}$ après fabrication, les fromages étaient enduits de « Pliol Holland » puis paraffinés.

\section{RESULTATS}

La technologie mise au point est présentée par le tableau 1.

Le tableau 2 montre la composition moyenne des laits utilisés.

Nous avons réalisé une standardisation en matière grasse de façon à obtenir un rapport protéine/matière grasse de 0,9 .

De forme cylindrique et d'un poids de $2 \mathrm{~kg}$, les fromages obtenus présentaient une pâte blanche, élastique, homogène et contenant quelques ouvertures. Le goût en était légèrement piquant et peu salé.

Le tableau 3 indique la composition des fromages obtenus dans les deux pays.

Les coefficients de transformation enregistrés sur les fromages affinés étaient les suivants :

- matière grasse : 85,6 en Grèce et 89,7 en Bulgarie ;

- protéines : 78,7 en Grèce et 81,7 en Bulgarie ;

- extrait sec : 52,9 en Grèce et 52 en Bulgarie.

A la lecture du tableau 3 , nous pouvons constater que, pour un taux d'humidité d'environ $39 \%$, le rendement était de $11 \%$.

Le tableau 4 présente l'évolution des différentes fractions azotées, au cours de l'affinage.

Le tableau 5 montre la composition en acides aminés libres d'un fromage après 3 mois d'affinage. Nous pouvons constater que le fromage est riche en acide glutamique, leucine, lysine et valine. 


\section{TABLEAU $1-T A B L E 1$}

Technologie d'un fromage semi-dur fait à partir du lait de chèvre Technology of a semi-hard cheese made from goat's milk

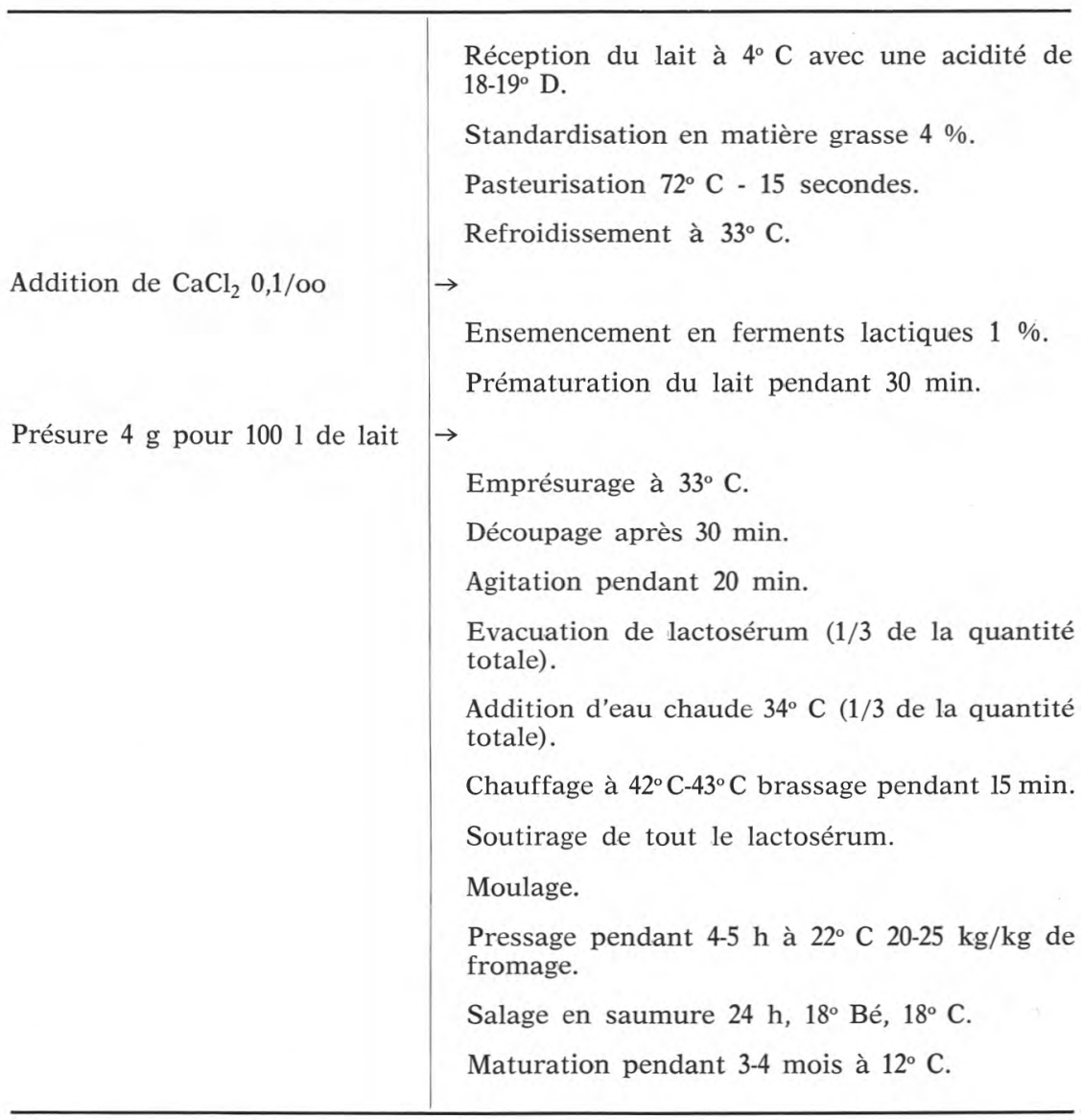

\section{CONCLUSION}

Nous sommes parvenus à fabriquer à partir de lait de chèvre un fromage de type Edam de qualité satisfaisante.

Après 3 mois d'affinage, la composition moyenne était la suivante : humidité : $39,2 \%$, taux de matière grasse sur extrait sec : $49 \%$. la haute teneur en acides aminés libres confère au fromage une grande valeur nutritive. 
TABLEAU 2 - TABLE 2

Composition moyenne du lait de chèvre utilisé pour la préparation du fromage Mean composition of goat's milk used for the preparation of cheese

\begin{tabular}{|c|c|c|c|c|c|c|c|c|c|c|}
\hline & $\begin{array}{l}\text { Mat. } \\
\text { grasse }\end{array}$ & $\begin{array}{l}\text { Prot. } \\
\text { totale }\end{array}$ & $\begin{array}{l}\text { Prot. } \\
\text { M. gras. }\end{array}$ & $\begin{array}{l}\text { Prot. } \\
\text { solub. }\end{array}$ & Caséine & $\begin{array}{l}\text { Cas. } \\
\text { M. gras. }\end{array}$ & Lactose & $\begin{array}{l}\text { Mat. } \\
\text { sèche }\end{array}$ & $\mathrm{pH}$ & Acidité \\
\hline $1^{\mathrm{e}}$ année & 4.73 & 3.74 & 0.79 & 0.79 & 2.92 & 0.61 & 4.74 & 13.42 & 6.44 & 0.19 \\
\hline $2^{\mathrm{e}}$ année & 4.0 & 3.67 & 0.92 & 0.72 & 2.96 & 0.74 & - & 13.06 & 6.40 & 0.18 \\
\hline $\begin{array}{l}\text { Position } \\
\text { deux stations }\end{array}$ & 4.90 & 3.67 & 0.75 & 0.92 & 2.71 & 0.55 & 4.31 & 13.65 & 6.54 & 0.17 \\
\hline
\end{tabular}


$T A B L E A U 3-T A B L E 3$

Composition moyenne du fromage - Mean composition of cheese

Bulgarie

\begin{tabular}{c|c|c|c|c|c|c|c|c|c|}
\hline jours & 44,68 & 25,44 & 46,0 & 24,28 & 3,73 & 15,36 & 0,9 & \\
mois & 42,69 & 26,90 & 46,9 & 24,89 & 5,08 & 20,41 & 1,75 & \\
mois & 41,75 & 28,10 & 48,2 & 26,21 & 7,68 & 29,30 & 2,45 & \\
mois & 39,16 & 29,80 & 49,0 & 26,87 & 8,37 & 31,15 & 2,68 & 11,06 & \\
mois & 38,75 & 30,20 & 49,3 & 27,95 & 8,90 & 31,84 & 2,85 & \\
\hline
\end{tabular}


TABLEAU 4 - TABLE 4

Composition de la matière azotée pendant la maturation du fromage Composition of the nitrogen matter of cheese during the ripening

\begin{tabular}{|c|c|c|c|c|c|c|}
\hline \multirow{2}{*}{ Sorte d'N } & & \multicolumn{5}{|c|}{ Age du fromage (jours) } \\
\hline & & 10 & 30 & 60 & 90 & 120 \\
\hline on soluble & $\%$ & 3,221 & 3,106 & 3,275 & 3,339 & 3,488 \\
\hline eptonique & $\%$ & 0,490 & 0,580 & 0,833 & 0,872 & 0,893 \\
\hline minique & $\%$ & 0,095 & 0,216 & 0,370 & 0,440 & 0,502 \\
\hline Total* & $\%$ & 3,806 & 3,902 & 4,108 & 4,211 & 4,381 \\
\hline
\end{tabular}

TABLEAU 5 - TABLE 5

Composition en acides aminés libres du fromage après affinage de 3 mois Composition in free aminoacids of cheese after 3 months of ripening

\begin{tabular}{l|c|c|c}
\hline Aminoacides & Mg/100 & Aminoacides & Mg/100 \\
\cline { 2 - 3 } Lys. & 266,98 & Ala. & 81,11 \\
His. & 103,19 & Cys. & - \\
Arg. & 11,28 & Val. & 239,96 \\
Asp. & 89,81 & Met. & 86,09 \\
Thr. & 117,46 & Ile. & 130,76 \\
Ser. & 94,73 & Leu. & 295,6 \\
Glu. & 520,16 & Tyr. & 30,76 \\
Pro. & 130,8 & Phe. & 132,54 \\
Gly. & 108,6 & & \\
\hline
\end{tabular}

Moyenne de 9 préparations. 


\section{Bibliographie}

Cogitore (A.) (1972). - Traité pratique de réglementation laitière. Edition du Sapin d'or, Epinal (France).

F.A.O./O.M.S. (1975). - Normes internationales recommandées pour les fromages et acceptations pour les gouvernements. (C.A.C.) C 1-C 25, p. 10. Edition F.A.O., Rome (Italie).

Dimitrov (D.), Obretenova (M.), ObRetenov (T.) (1976). - Etude sur l'arôme de Kaskaval. Les travaux scientifiques de l'industrie alimentaire, Plovdiv, I, $\mathrm{XX}$, III, part II.

F.I.L. - Int. standard, $\mathrm{n}^{\text {os }}$ 17-1961, 20-1962, 21-1961, 29-1964, 28-1968.

Schneider (Sc.) (1954). - Traité pratique des essais du lait et de contrôle de produits laitiers. Imp.-éd. Les Hoirs, Wyss (G. Z.), Berne (Suisse).

Veinoglou (B.) (1976). - Fromagerie. Cours pour les étudiants, p. 143, Athènes (Grèce).

Veinoglou (B.), Baltadjieva (M.), Kalatzopoulos (G.) Dalles (T.) et Stamenova (V.) (1982). - La préparation des fromages à pâte molle à partir du lait de chèvre. Recherche Agricole, 1983 (sous presse).

Veinoglou (B.), Baltadjieva (M.), Kalatzopoulos (G.), Stamenova (V.) et PapadoPoulou (E.) (1982). - Composition du lait de chèvre de la région de Plovdiv en Bulgarie et de Ioannina en Grèce. Le Lait, 62, 613-614, 155-165. 